\title{
FUNCTION ALGEBRAS WITH CLOSED RESTRICTIONS ${ }^{1}$
}

\section{GLICKSBERG}

Let $C(X)$ be the (supremum normed) algebra of all complex continuous functions on a compact Hausdorff space $X$, and $A$ a closed subalgebra containing the constants and separating the points of $X$. For $F \subset X$ let $f \mid F$ be the restriction of the function $f$ to $F, A \mid F$ $=\{f \mid F: f \in A\}$. The purpose of this note is to prove the following result, which characterizes $C(X)$ among its closed separating subalgebras.

THEOREM. Suppose that for every closed $F \subset X, A \mid F$ is closed in $C(F)$. Then $A=C(X)$.

Our proof leans heavily on some facts obtained recently by Katznelson [1]. In fact little else, beyond the Riesz representation theorem, is needed (for some facts concerning Banach algebras the reader is referred to $[2 ; 3])$.

Let $F$ be a closed subset of $X . k F=\{f: f \in A, f(F)=0\}$, the kernel of $F$, is an ideal in $A$, and we can form the quotient algebra $A / k F$; for an element $f+k F(f \in A)$ of $A / k F,\|f+k F\|$ will denote the usual quotient norm (inf $\{\|f+g\|: g \in k F\}$ ) under which $A / k F$ is a Banach algebra. For $x \in F, f+k F \rightarrow f(x)$ is a multiplicative linear functional on $A / k F$, so necessarily of norm $\leqq 1$, whence $\| f|F||=\sup | f(F) \mid$ $\leqq\|f+k F\|$, where $\|f \mid F\|$ is of course the norm inherited by the closed subalgebra $A \mid F$ of $C(F)$. Now clearly $f+k F \rightarrow f \mid F$ is an isomorphism of $A / k F$ onto $A \mid F$, and so, by the open mapping theorem, topological: there is a constant $c_{F}$ for which

$$
\|f+k F\| \leqq c_{F}\|f \mid F\|, \quad f \in A,
$$

a relation which we shall need somewhat later.

To begin the proof proper we shall show that for closed disjoint subsets $F$ and $K$ of $X$ there is an $f$ in $A$ which is 1 on $F, 0$ on $K$. Indeed let $x$ and $y$ be distinct elements of $X$. Since $A$ separates $X$, there is an $f$ in $A$ with $f(x)=0, f(y)=1$, so we can find closed neighborhoods $V_{x}$ of $x$ and $W_{x}$ of $y$ for which $\left|f\left(V_{x}\right)\right| \leqq \frac{1}{4},\left|1-f\left(W_{x}\right)\right| \leqq \frac{1}{4}$. As is well known there is a sequence $\left\{p_{n}\right\}$ of polynomials in one complex variable which converge uniformly on $\left\{z:|z| \leqq \frac{1}{4}\right\} \cup\left\{z:|1-z| \leqq \frac{1}{4}\right\}$ to the characteristic function of $\left\{z:|z| \leqq \frac{1}{4}\right\}$; consequently

Received by the editors January 5, 1962.

1 Work supported in part by the National Science Foundation through Grant G14779, and in part by the Air Force Office of Scientific Research. 
$\left\{\left(p_{n} \circ f\right) \mid\left(V_{x} \cup W_{x}\right)\right\}$ is a sequence of elements of $A \mid\left(V_{x} \cup W_{x}\right)$ which converges uniformly on $V_{x} \cup W_{x}$, tending to 1 on $V_{x}, 0$ on $W_{x}$. Since $A \mid\left(V_{x} \cup W_{x}\right)$ is closed under uniform convergence by hypothesis, there is an $e$ in $A$ which is 1 on $V_{x}, 0$ on $W_{x}$.

Now let $y$ be a fixed element of $K$. $F$ can be covered by finitely many $V_{x}, x \in F$, say $V_{x_{1}}, \cdots, V_{x_{n}}$. If $e_{1}, \cdots, e_{n}$ are the corresponding elements of $A$, then (as in a familiar argument) clearly $e_{1}^{\prime}=e_{1}+e_{2}$ $-e_{1} e_{2}$ is 1 on $V_{x_{1}} \cup V_{x_{2}}$ and 0 on the neighborhood $W_{x_{1}} \cap W_{x_{2}}$ of $y$; similarly $e_{2}^{\prime}=e_{1}^{\prime}+e_{3}-e_{1}^{\prime} e_{3}$ is 1 on $V_{x_{1}} \cup V_{x_{2}} \cup V_{x_{3}}$ and 0 on $W_{x_{1}} \cap W_{x_{2}}$ $\cap W_{x_{3}}$. Continuing we thus obtain an element $f_{v}$ of $A$ which is 1 on $F$, and vanishes on some neighborhood $U_{y}$ of $y$.

But now finitely many $U_{v}$ cover $K$, so that the product $f$ of the corresponding $f_{\nu}$ yields an element of $A$ which is 1 on $F$ and 0 on $K$, as desired.

Alternatively stated, we have found an idempotent in $A / k(K \cup F)$ which separates $F$ and $K$. What we now require is a bound on the norms of such idempotents, and to obtain this we repeat an argument of Katznelson's [1, Lemmas 3, 4, 5]. Let us say $A$ is bounded on a subset $V$ of $X$ if there is a constant $C_{V}$ for which, whenever $F$ is closed and $F \subset V$, any idempotent in $A / k F$ has norm $<C_{\nabla}$. (Our next lemmas are taken from Katznelson [1], and are repeated here for the convenience of the reader.)

Lemma 1. Let $V_{1}$ and $V_{2}$ be open in $X$, and $A$ be bounded on each $V_{i \text {. }}$ Then $A$ is bounded on every closed subset $F$ of $V_{1} \cup V_{2}$.

Proof. Since $F \backslash V_{2}$ is a closed subset of $V_{1}$ we can find an open $W_{1} \supset F \backslash V_{2}, W_{1}^{-} \subset V_{1}$; and since $F \backslash W_{1} \subset V_{2}$ there is an open $W_{2} \supset F \backslash W_{1}$, $W_{2}^{-} \subset V_{2} . F \backslash W_{1}$ and $F \backslash W_{2}$ are disjoint closed subsets of $X$ so there is a $\phi$ in $A$ for which $\phi\left(F \backslash W_{1}\right)=0, \phi\left(F \backslash W_{2}\right)=1$, as we have seen.

Let $P$ be a closed subset of $F$ and let $f+k P$ be an idempotent in $A / k P$, so $f$ is zero or 1 at each point of $P$. Then $f+k\left(P \cap W_{i}^{-}\right)$is an idempotent in $A / k\left(P \cap W_{i}\right)$ and so (by the definition of the quotient norm) we can choose $f_{i} \in f+k\left(P \cap W_{i}^{-}\right)$with $\left\|f_{i}\right\|<C_{V_{i}}, P \cap W_{i}^{-}$being a closed subset of $V_{i}, i=1,2$. But now $f=\phi f_{1}+(1-\phi) f_{2}$ on $P$, since $P \subset F \subset W_{1} \cup W_{2}$, and thus

$$
\|f+k P\| \leqq\left\|\phi f_{1}+(1-\phi) f_{2}\right\|<\|\phi\| C_{V_{1}}+\|1-\phi\| C_{V_{2}}
$$

so the right side may be taken as $C_{F}$.

Still following Katznelson let us say $A$ is bounded at $x \in X$ if $A$ is bounded on $V$, for some neighborhood $V$ of $x$. Then as an obvious consequence of Lemma 1 and compactness we have

Lemma 2. If $F$ is closed and $A$ is bounded at each $x$ in $F$ there is an 
open $V \supset F$ on which $A$ is bounded.

LEMMA 3. There are at most finitely many $x$ in $X$ at which $A$ is not bounded.

Proof. If there were infinitely many we could find a sequence $\left\{x_{n}\right\}$ of them with disjoint open neighborhoods $\left\{V_{n}\right\}$ for which $A$ is not bounded on $V_{n}$. So each $V_{n}$ would contain a closed $F_{n}$ for which there is an idempotent $f_{n}+k F_{n}$ in $A / k F_{n}$ of norm $\geqq n$.

Now let $F=\left(U F_{n}\right)^{-}$; since $V_{n} \cap F_{m}=\varnothing$ for $n \neq m, F=F_{n}$ $\cup\left(\bigcup_{m \neq n} F_{m}\right)^{-}, F_{n} \cap\left(\bigcup_{m \neq n} F_{m}\right)^{-}=\varnothing$ and thus there is a $\phi$ in $A$ with $\phi\left(F_{n}\right)=1, \phi\left(F \backslash F_{n}\right)=0$. Consequently $\phi f_{n}$ is zero or 1 at each $x$ in $F$, and $\phi f_{n}+k F$ is an idempotent in $A / k F$; but

$$
n \leqq\left\|f_{n}+k F_{n}\right\|=\left\|\phi f_{n}+k F_{n}\right\| \leqq\left\|\phi f_{n}+k F\right\|
$$

(since $k F \subset k F_{n}$ ) so the idempotents in $A / k F$ are not bounded in norm. By (1) they must be bounded, and the lemma is proved.

Let $\left\{x_{1}, \cdots, x_{n}\right\}$ be the finite set given by Lemma 3 and let $F$ be any closed set in $X$ disjoint from $\left\{x_{1}, \cdots, x_{n}\right\}$. By Lemma 2, $A$ is bounded on a neighborhood of $F$; so there is a constant $C_{F}$ for which, whenever $K$ is closed, $K \subset F$, each idempotent in $A / k K$ has norm $<C_{F}$.

Now suppose $\mu$ is a (complex regular Borel) measure on $F$ orthogonal to the closed subalgebra $A \mid F$ of $C(F)$, and let $K$ be a closed subset of $F, \epsilon>0$. Choose a closed $K_{0} \subset F \backslash K$ for which the total variation of $\mu$ on $(F \backslash K) \backslash K_{0}$ is less than $\epsilon / C_{F}$, and then choose $f \in A$ which is 1 on $K, 0$ on $K_{0}$, and of norm $<C_{F}$. Then

$$
0=\int f d \mu=\int_{K} 1 d \mu+\int_{K_{0}} 0 d \mu+\int_{(F \backslash K) \backslash K_{0}} f d \mu,
$$

so $|\mu(K)|=\left|\int(F \backslash K) \backslash K_{0} f d \mu\right| \leqq C_{F} \cdot \epsilon / C_{F}=\epsilon$. Thus since $\epsilon>0$ is arbitrary, $\mu K=0$ for every closed $K \subset F$, and $\mu$ itself vanishes by regularity. Consequently $A \mid F=C(F)$.

Now this implies any measure $\mu$ orthogonal to $A$ is carried by $\left\{x_{1}, \cdots, x_{n}\right\}$. For if not $\mu F \neq 0$ for some closed $F$ disjoint from $\left\{x_{1}, \cdots, x_{n}\right\}$ by regularity, and if $V$ is an open neighborhood of $\left\{x_{1}, \cdots, x_{n}\right\}$ with $V^{-} \cap F=\varnothing$, we can choose $f \in A$ with $f\left(V^{-}\right)=0$, $f(F)=1$; then $f \mu$ (the measure corresponding to the functional $g \rightarrow \int g f d \mu$ on $\left.C(X)\right)$ is a measure carried by $X \backslash V$, and orthogonal to $A$ and so orthogonal to $A \mid(X \backslash V)=C(X \backslash V)$, hence the zero measure. But $f \mu(F)=\int_{R} f d \mu=\mu F \neq 0$, the desired contradiction, and $\mu$ orthogonal to $A$ must be carried by $\left\{x_{1}, \cdots, x_{n}\right\}$. 
Since $A$ separates the points of $X$ this is clearly impossible unless all orthogonal measures vanish, i.e., unless $A=C(X)$, which completes the proof of the theorem.

\section{REFERENCES}

1. Y. Katznelson, $A$ characterization of the algebra of all continuous functions on a compact Hausdorff space, Bull. Amer. Math. Soc. 66 (1960), 313-315.

2. L. H. Loomis, Abstract harmonic analysis, Van Nostrand, New York, 1953.

3. C. Rickart, General theory of Banach algebras, Van Nostrand, New York, 1960.

INSTITUTE FOR AdVANCEd STUdY 